\title{
An EMT-DP Hybrid Simulation Method of AC-DC Power System
}

\author{
Gang Luo ${ }^{1}$, Chongru Liu ${ }^{2}$, Wei Xu ${ }^{2, *}$, Yinguo Yang ${ }^{1}$, Feng Qian ${ }^{1}$ and Qingang Duan ${ }^{1}$ \\ ${ }^{1}$ Guangdong Power Grid Compang Limited, China \\ ${ }^{2}$ North China Electric Power University, China \\ ${ }^{*}$ Corresponding author
}

\begin{abstract}
In order to take the advantage of electromagnetic transient (EMT) simulation method and dynamic phasors simulation method, a new hybrid simulation of AC-DC system, electromagnetic transient - dynamic phasors hybrid simulation of AC-DC system, where dynamic phasors model and traditional EMT models are applied to build the model of HVDC system and AC system respectively is proposed. The location of the interface, the mutual equivalent method between $\mathrm{AC}$ and DC system in the proposed hybrid simulation and the simulation process of the proposed hybrid simulation are investigated. Simulation results show that the proposed electromagnetic transient - dynamic phasors hybrid simulation of AC-DC systems are effective and acceptable.
\end{abstract}

Keywords- AC-DC system; dynamic phasors; electromagnetic transient; hybrid simulation

\section{INTRODUCTION}

With the improvement of power electronics devices and the demand of large-capacity long-distances power transmission over, high-voltage direct current transmission technology is widely used in the world. Especially in China, where the distance between the center of power and load is farther, HVDC plays an important role in the regional interconnection of power systems [1]. To analyze the dynamic process of ACDC system, the researchers have focused on simulation model and method of AC-DC systems for different purposes and different levels of detail.

The simulation models used widely in the domain simulation of power system can be divided into two classes: electromagnetic transient simulation model [2,3] and electromechanical transient simulation model [4]. The electromagnetic transient model based on the differential model can reflect the frequency dynamics of AC-DC systems accurately. However, its requisite calculating resource is very large, especially the electromagnetic transient simulation of power electronic devices. Electromechanical transient (EMT) simulation model adopting the fundamental phasor model can reduce the calculating scale, while its accuracy is less than ideal as DC converters and AC systems are simulated as conventional quasi-steady model.

To solve the problem between the simulation precision and computing scale, dynamic phasors simulation theory [5] is introduced to the modelling of power system due to its advantages in modelling of power electronic device. The theory is to find out the time-varying Fourier coefficient of the principal components of the time-varying signal based on Fourier transform, and then build dynamic phasors models which could reflect dynamic characteristics of the specified component. This method has utilized in modelling for power electronics successfully, such as HVDC systems [6,7], FACTS [8] and DC - DC converter [9]. To take advantage of these three models in AC-DC system simulation, dynamic phasor simulation, electromechanical transient - dynamic phasor simulation and electromagnetic transient - dynamic phasor simulation of AC-DC system have been researched in [10-12].

The EMT model of the AC system can use only one simulation model to reflect the full dynamic characteristics of the fundamental and all harmonics components of AC systems, and dynamic phasor model of DC system can build the algebraic mathematical model of the main characteristic harmonic of converter based on the need of simulation accuracy to reduce the computational complexity of the modelling and simulation. In order to take the advantage of EMT simulation method and dynamic phasors simulation method, a new hybrid simulation of AC-DC system, electromagnetic transient - dynamic phasors (EMT - DP) hybrid simulation of AC-DC system, where dynamic phasors model and traditional EMT models are applied to build the model of HVDC system and AC system respectively is proposed, and the dynamic phasors model of HVDC system proposed in [7] is used.

The paper is organized as follows: Section 2 introduces the basic principle of EMT simulation model and dynamic phasors model. Section 3 analyses the partition and mutual equivalent method of AC-DC system, and the simulation process of EMTDP hybrid simulation of AC-DC system. Section 4 shows the simulation results and Section 5 draws the conclusions of this paper.

\section{The BASIC PRINCIPLE OF EMT SiMULATION MODEL AND DYNAMIC PHASORS MODEL}

\section{A. The Basic Principle of EMT Simulation Model}

EMT simulation is based on the implicit trapezoidal integral method of differential equation and can reflect the dynamic characteristics of components equipped in AC system, such as inductors, power filters and transmission lines, as the implicit trapezoidal integral method has a very high accuracy and computational stability in solving the differential equation. 
Take the inductance element as an example to describe the numerical calculation method of EMT simulation. The time domain differential equation of inductor is,

$$
L \frac{d i_{L}}{d t}=u_{L}
$$

where $u_{\mathrm{L}}$ and $i_{\mathrm{L}}$ are the voltage across inductor and the current flowing through inductor respectively. Using the implicit trapezoidal integral method, the above equation can be transformed into the following difference equation.

$$
i(t+\Delta t)=\frac{\Delta t}{2 L}\left[u_{L}(t+\Delta t)-u_{L}(t)\right]+i(t)
$$

where $\Delta t$ is the integral solution step.

The simulation processes of EMT simulation of AC system are described in [13].

\section{B. The Basic Principle of Dynamic Simulation Model}

The mathematical basis of the dynamic phasors model is the Fourier transform. For a signal, $x(\tau)$, with a period $\mathrm{T}$ in the time domain, its Fourier series in the interval of $(\mathrm{t}-\mathrm{T}, \mathrm{t}] \mathrm{can}$ be expressed as follows .

$$
\begin{gathered}
x(\tau)=\sum_{k=-\infty}^{\infty} X_{k}(t) e^{j k \omega \tau} \\
X_{k}(t)=\frac{1}{T} \int_{t-T}^{t} x(\tau) e^{-j k \omega \tau} d \tau
\end{gathered}
$$

Where $\omega=2 \pi / \mathrm{T} ; X_{\mathrm{k}}(t)$ is the $k$ th time-variant Fourier coefficient and called k-order dynamic phasor, recorded as $\langle x\rangle_{k}$.

According to the basis of dynamic phasors theory, the conversion from time domain value to dynamic phasors value can be completed as follows.

$$
\begin{aligned}
& \langle X\rangle_{k}(t)=\frac{1}{T} \int_{t-T}^{t} x(\tau) \mathrm{e}^{-j k\left(\frac{2 \pi}{T}\right) \tau} d \tau \\
& =\frac{1}{N} \sum_{n=1}^{N} x(t-T+n \Delta t) e^{-j k \frac{2 \pi}{T}(t-T+n \Delta t)}
\end{aligned}
$$

where $N=T / \Delta t, \Delta t$ is the integral solution step.

According to the definition of the $k$-order dynamic phasor, the dynamic phasors have the following characteristics.

1) Conjugate property:

$$
\langle x\rangle_{-k}=\langle x\rangle_{k}^{*}
$$

where "*" indicates the conjugate complex number.

2) Differential property:

$$
\frac{d\langle x\rangle_{k}}{d t}=\left\langle\frac{d x}{d t}\right\rangle_{k}-j k \omega\langle x\rangle_{k}
$$

3) Convolution property:

$$
\left\langle x_{1} x_{2}\right\rangle_{k}=\sum_{i=-\infty}^{\infty}\left\langle x_{1}\right\rangle_{k-i}\left\langle x_{2}\right\rangle_{i}
$$

By using the dynamic phasors theory and the modulation theory of the DC system converter, it is convenient to establish the dynamic phasors model of the converter which can reflect the dynamic characteristics of the converter. Besides, the dynamic phasors differential model of DC transmission line and the DC system controller could be established..

\section{The EMT-DP Hybrid SimUlation METHOD OF AC- DC SYSTEM}

In the proposed hybrid simulation method, the traditional EMT simulation method is applied to build the model of AC system and the dynamic phasors model of HVDC system proposed in [7], including the dynamic phasor models of the converter, the DC line, the constant current and constant voltage controllers and the voltage dependent current order limiter (VDCOL), is used to build the model of HVDC system.. However, the dynamic phasor model of HVDC system proposed in [7] doesn't establish the model of the phase-locked loop (PLL). In order to realize the hybrid simulation and improve the model of DC system control system, the model of the PLL is established based on [14].

\section{A. The Partition and the Equivalent Method of AC-DC System}

The commutation buses at the rectifier side and the inverter side are selected as the interface of AC and DC system in the proposed hybrid simulation. According to the interrelationship between AC and DC system, the partition of $\mathrm{AC}$ and DC system simulation and the location of interface in the simulation processes, $\mathrm{AC}$ system is equivalent as a voltage source to the DC system at converter buses, and DC system is equivalent as current source to $\mathrm{AC}$ system at the converter bus. In addition, due to the DC side filter inductance, the DC side of DC system takes the direct current component into account. The current at AC side considers the fundamental current and the main harmonic currents according to the form of the converter. Taking the 12-plus converter as example, the fundamental current as well as the 11 and 13 harmonic currents should be considered at AC side.

\section{B. The Process of EMY-DP Hybrid Simulation of AC-DC System}

The EMT-DP simulation method are as follows. Firstly, an EMT simulation network of AC system is established according to the position of AC and DC system. Then get dynamic phasor models of the DC line, the controller and the 
PLL model differentiated. Finally, the EMT simulation network of AC system dynamic phasors model of the converter and the differential dynamic phasor model of DC line, controllers and the PLL cooperate with each other to achieve the hybrid simulation. The simulation flowchart is shown in Figure 1.

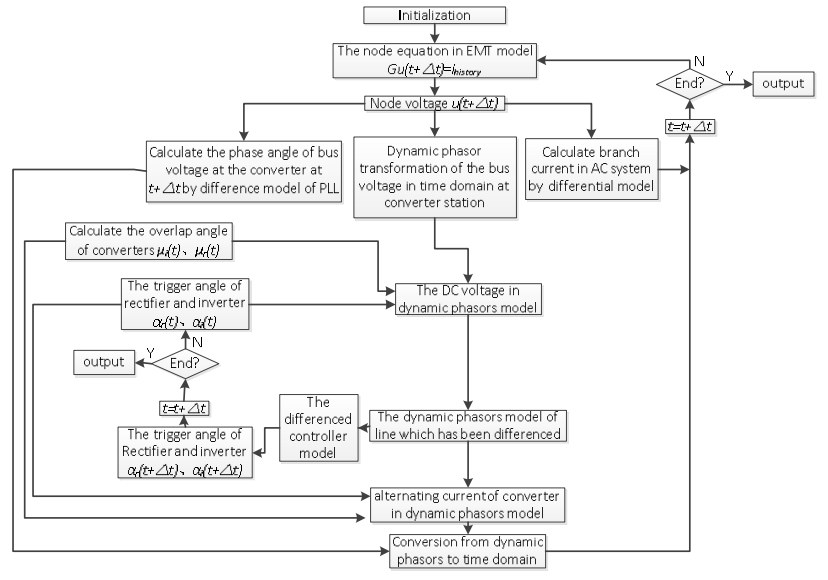

FIGURE I. THE FLOWCHART OF EMT-DP HYBRID SIMULATION OF AC-DC SYSTEM

Step 1The commutation buses at the rectifier and inverter sides are taken as the boundary. The node admittance equations of the EMT simulation network of AC system are shown as formula 8 according to the connection relationships of the components in AC system, such as filter, and the position of DC system.

$$
G u_{a c}(t+\Delta t)=i_{\text {history }}(t)
$$

where $G$ is the node admittance matrix of the EMT simulation formed by components of AC system, $u_{a c}(t+\Delta t)$ is the column vector of instantaneous voltage of each node in AC system, $i_{\text {history }}(t)$ is the column vector of historical current composed of the voltage value of each node, the current value of each branch, and the time domain value of the AC current flowing from converter buses to DC system.

Step 2 According to the formula (8), the instantaneous voltage values of each node at $u_{a c}(t+\Delta t)$ moment are obtained, recorded as $u_{a c}(t+\Delta t)$.

Step 3 According to the differential model of component $\mathrm{s}$ of AC system, the current values of each branch at $t+\Delta t$ moment are obtained, recorded as $i_{a c}(t+\Delta t)$.

Step 4 Sending the AC voltage values of the converter bus at $t$ and $t+\Delta t$ moments and the phase angles of the voltages of Phase A of the converter bus at the $t$ moment, $\theta_{r a}(t)$ and $\theta_{i a}(t)$, to the differential model of the PLL to obtain the phase angles of the converter bus voltage at the rectifier and inverter stations at $t+\Delta t$ moment, $\theta_{r a}(t+\Delta t)$ and $\theta_{i a}(t+\Delta t)$.

Step 5 By formula (5), the dynamic phasors values of the voltages of phase A of the converter bus at the rectifier and inverter side at $t+\Delta t$ moment are obtained, recorded as $\left\langle v_{r a}\right\rangle_{1}(t+\Delta t)$ and $\left\langle v_{i a}\right\rangle_{1}(t+\Delta t)$.

Step 6 Sending the dynamic phasors value of voltage of Phase A of the converter bus at both sides at $t+\Delta t$ moment, $\left\langle v_{r a}\right\rangle_{1}(t+\Delta t)$ and $\left\langle v_{i a}\right\rangle_{1}(t+\Delta t)$, the outputs of the controllers at $t$ moment, $\alpha_{r}(t)$ and $\alpha_{i}(t)$, and the commutation angles of the rectifier and inverter at $t$ moment calculated by the dynamic phasors model of HVDC system proposed in [7], $\mu_{r}(t)$ and $\mu_{i}(t)$, to DC voltage parts of dynamic phasors models of converter proposed in [7] to obtain dynamic phasors values of DC voltages at the rectifier and inverter sides at $t+\Delta t$ moment, $\left\langle v_{d r}\right\rangle_{0}(t+\Delta t)$ and $\left\langle v_{d i}\right\rangle_{0}(t+\Delta t)$.

Step 7 Sending the dynamic phasor values of DC voltages at $t+\Delta t$ moment, $\left\langle v_{d r}\right\rangle_{0}(t+\Delta t)$ and $\left\langle v_{d i}\right\rangle_{0}(t+\Delta t)$, the dynamic phasor values of DC voltages at $t$ moment, $\left\langle v_{d r}\right\rangle_{0}(t)$, $\left\langle v_{d i}\right\rangle_{0}(t)$ and $\left\langle v_{d c}\right\rangle_{0}(t)$, and the dynamic phasor values of DC currents at $t$ moment, $\left\langle i_{d r}\right\rangle_{0}(t),\left\langle i_{d i}\right\rangle_{0}(t)$ and $\left\langle i_{d c}\right\rangle_{0}(t)$, to the differential dynamic phasor model of DC line to obtain the dynamic phasors values of DC currents, $\left\langle i_{d r}\right\rangle_{0}(t+\Delta t)$, $\left\langle i_{d i}\right\rangle_{0}(t+\Delta t)$ and $\left\langle i_{d c}\right\rangle_{0}(t+\Delta t)$, and the dynamic phasors value of voltage across the capacitor, $\left\langle v_{d c}\right\rangle_{0}(t+\Delta t)$.

Step 8 Sending the dynamic phasors values of the DC voltages and currents at $t+\Delta t$ time, is $\left\langle v_{d r}\right\rangle_{0}(t+\Delta t),\left\langle v_{d c}\right\rangle_{0}(t+\Delta t),\left\langle v_{d i}\right\rangle_{0}(t+\Delta t) \quad$ and $\left\langle i_{d r}\right\rangle_{0}(t+\Delta t),\left\langle i_{d i}\right\rangle_{0}(t+\Delta t),\left\langle i_{d c}\right\rangle_{0}(t+\Delta t), \quad$ to the transformation formula from dynamic phasors values to time domain values to obtain the time domain values of DC voltages and currents, $v_{d r}(t+\Delta t), v_{d c}(t+\Delta t), v_{d i}(t+\Delta t)$ and $i_{d r}(t+\Delta t), i_{d c}(t+\Delta t), i_{d i}(t+\Delta t)$.

Step 9 Sending the dynamic phasors values of DC currents at $t+\Delta t$ moment, $\left\langle i_{d r}\right\rangle_{0}(t+\Delta t)$ and $\left\langle i_{d i}\right\rangle_{0}(t+\Delta t)$, the firing angles of the rectifier and inverter at $t$ moment, $\alpha_{r}(t)$ and $\alpha_{i}(t)$, the commutation angles of the rectifier and inverter at $t$ moment calculated by the dynamic phasors model of HVDC system proposed in [7], $\mu_{r}(t)$ and $\mu_{i}(t)$, the phase angles of voltages of Phase A of commutation buses at the rectifier and inverter side at $t+\Delta t$ moment calculated by the model of the PLL, $\theta_{r a}(t+\Delta t)$ and $\theta_{i a}(t+\Delta t)$, and the initial phase angles of the voltage of Phase A of converter buses at rectifier and inverter side to the AC current parts of dynamic phasors models of converters and the transformation from dynamic phasors values to time domain values proposed in [7] to obtain the values of AC current flowing from commutation bus to the HVDC system at the rectifier and inverter side at $t+\Delta t$ moment, $i_{r a}(t+\Delta t), i_{r b}(t+\Delta t), i_{r c}(t+\Delta t)$ and $i_{i a}(t+\Delta t), i_{i b}(t+\Delta t), i_{i c}(t+\Delta t)$.

Step 10 Sending the dynamic phasors values of the voltage and the current at the DC side at $t+\Delta t$ moment, $\left\langle v_{d i}\right\rangle_{0}(t+\Delta t)$, $\left\langle i_{d r}\right\rangle_{0}(t+\Delta t),\left\langle i_{d i}\right\rangle_{0}(t+\Delta t)$, and the dynamic phasors values of the voltage and the currents at the DC side at $t$ moment, 
$\left\langle v_{d i}\right\rangle_{0}(t),\left\langle i_{d r}\right\rangle_{0}(t),\left\langle i_{d i}\right\rangle_{0}(t)$, to the differential model of controllers to obtain the firing angles of the rectifier and inverter at $t+\Delta t$ moment, $\alpha_{r}(t+\Delta t)$ and $\alpha_{i}(t+\Delta t)$.

Step 11 Make $t=t+\Delta t$. If $t=t \_e n d$, the simulation is over; if not, go back to step 1 .

\section{The Simulation Results}

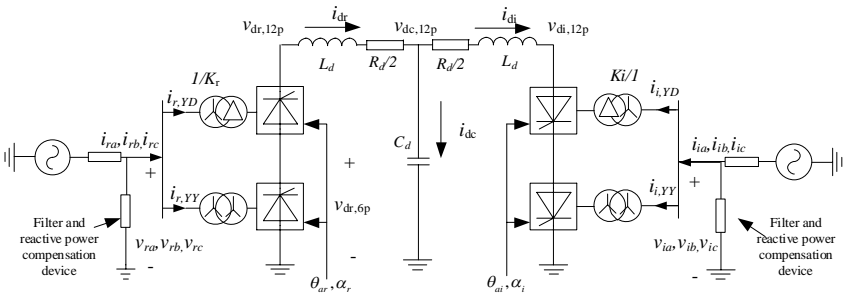

FIGURE II. A MONOPOLAR 12-PULES HVDC SYSTEM

The simulation test system is a monopolar 12-pulse HVDC system shown in figure 2. Parameters of the test system are as follows: the frequency of $\mathrm{AC}$ system is $50 \mathrm{~Hz}$, the phase-tophase RMS voltage of source in the AC system at rectifier side is $382.8672 \mathrm{kV}$, the converter transformer ratio in rectifier station is $1 / K r=345 / 213.4557$, the phase-to-phase RMS voltage of source in the AC system at inverter side is $215.05 \mathrm{kV}$, the converter transformer ratio in inverter side is $1 / \mathrm{Ki}=230 / 209.2288$, the capacities of the converter transformers at the rectifier and the inverter side are 603.73MW and 591.79MVA respectively, the leakage reactances of the transformers at the rectifier side and the inverter side are $0.18 \mathrm{pu}$, and 11th harmonic filters, 13th harmonic filters and reactive power compensation devices are equipped at the rectifier and inverter side.

The rated voltage and rated current of HVDC system are $500 \mathrm{kV}$ and $2 \mathrm{kA}$ respectively. the resistance of the DC transmission line is $5 \Omega$; the inductances of the smooth reactors at the rectifier side and the inverting side of the DC line are $0.5968 \mathrm{H}$ respectively, and the capacitance in the middle of the DC line is $50 \mathrm{uF}$. The constant current control and constant voltage control are used at the rectifier and inverter sides respectively and voltage dependent current order limiters are equipped at the rectifier and inverter sides respectively.

The EMT simulation model of AC-DC system shown in Figure 2 is built in PSCAD/EMTDC. With the proposed EMT-DP hybrid simulation method, the hybrid simulation of the AD-DC system shown in Figure 2 is implemented in MATLAB, where the simulation steps of the EMT simulation and the proposed hybrid simulation are $50 \mu \mathrm{s}$ and $100 \mu \mathrm{s}$ respectively. The simulation results got by hybrid simulation have been compared with the simulation results got by the EMT simulation in the following two kinds of disturbances.

Case 1: The reference value of DC current in HVDC

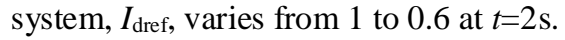

Case 2: The AC voltages of the source at the rectifier side drop $5 \%$ at $t=2 \mathrm{~s}$, which is usually caused by a distant fault ${ }^{[10]}$

Under these two kinds of disturbance, the simulation results of the DC current at rectifier side got by the proposed hybrid simulation and EMT simulation are shown in Figure 3; the simulation results of Phase A voltage of converter bus at rectifier side got by these two simulations are shown in Figure 4; the simulation results of AC current of phase A flowing from the commutation bus to DC system at the rectifier side ate shown in Figure 5, where $i_{d r H y b r i d}, v_{\text {raHybrid }}, i_{\text {raHybrid }}$ are the DC current, AC voltage and AC current got by the proposed hybrid simulation respectively, $i_{d r E M T}, v_{\text {raEMT }}, i_{\text {raEMT }}$ are the DC current, AC voltage and AC current got by the EMT simulation respectively.

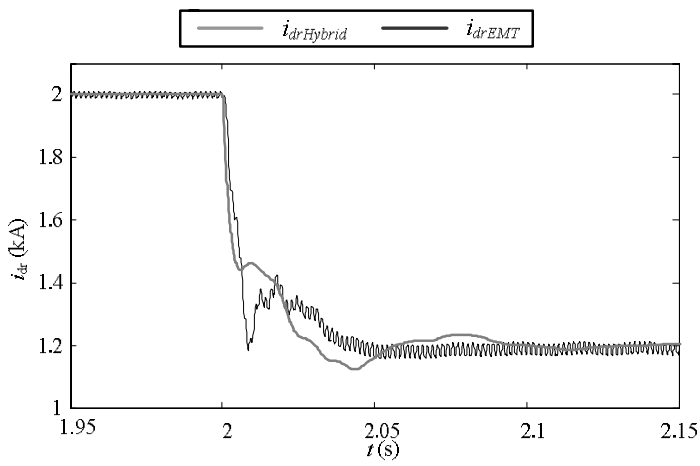

(a) Case 1

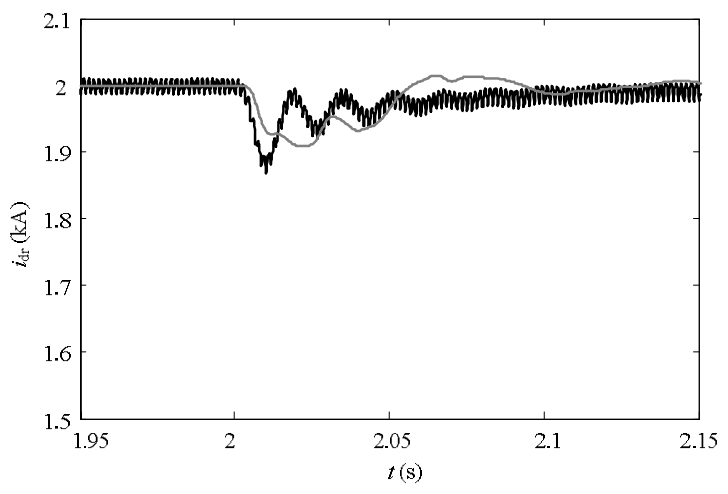

(b) Case (2)

FIGURE III. SIMULATION RESULTS OF DIRECT CURRENT

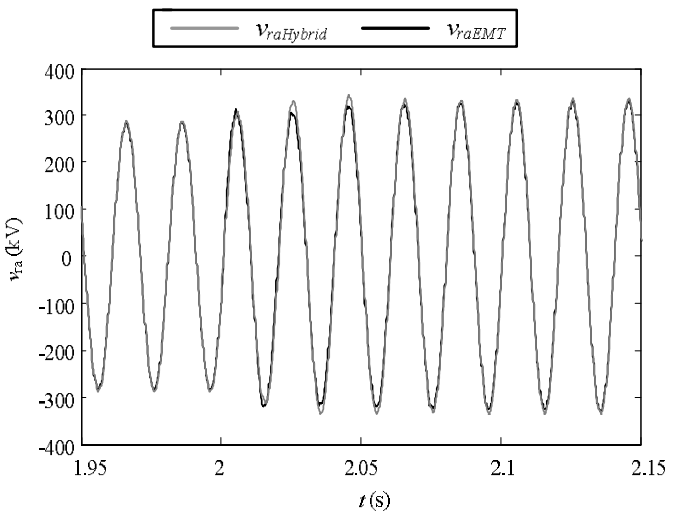

(a) Case (1) 


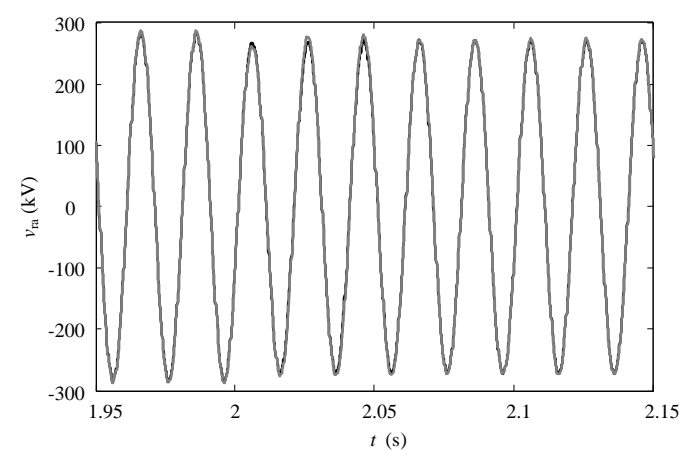

(b) Case (2)

FIGURE IV. SIMULATION RESULTS OF COMMUTATION BUS VOLTAGE IN RECTIFIER SIDE

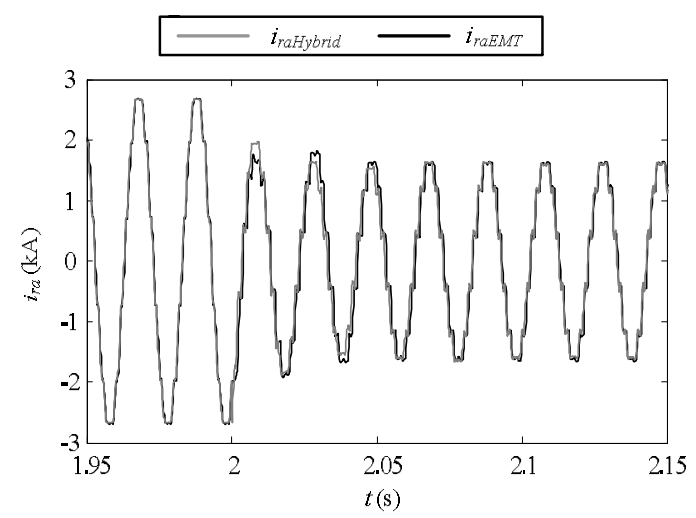

(a) Case 1

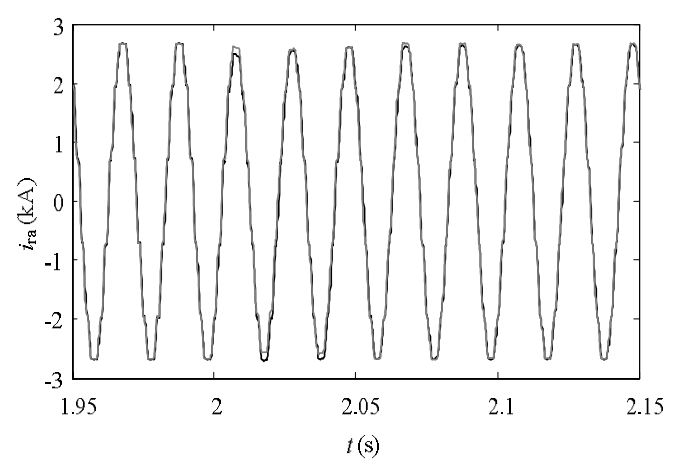

(b) Case 2

FIGURE V. SIMULATION RESULTS OF AC CURRENT IN RECTIFIER SIDE

Simulation results show that the EMT-DP hybrid simulation can reflect the dynamic characteristics of AC-DC system including the harmonic characteristics of HVDC converter under the disturbance from DC system and AC system.

What's more, the simulation step of electromagnetic transient model is $50 \mu \mathrm{s}$, while the simulation step of the proposed hybrid model is $100 \mu \mathrm{s}$. Because the EMT model and the proposed hybrid simulation model are established on different platforms, the simulation speed can not be compared directly. But the simulation step of the proposed hybrid simulation is larger than that of the EMT simulation. On the other hand, the dynamic phasor modelling method can establish the converter model as algebraic equations to reflect the frequency characteristics of converter accurately. Therefore, compared with the electromagnetic transient calculation method, the proposed hybrid simulation method can reduce the computational scale effectively. At the same time, the dynamic phasors simulation method can reduce simulation time effectively is analysed in detail in [6]. As a consequence, the proposed hybrid simulation method can reflect the dynamic characteristics of AC-DC systems effectively improve the simulation speed.

\section{CONCLUSION}

In order to take the advantage of EMT simulation method and dynamic phasors simulation method, a new hybrid simulation method of AC-DC power system, electromagnetic transient - dynamic phasors hybrid simulation of AC-DC system, is proposed, where dynamic phasors model and traditional EMT models are applied to build the model of HVDC system and AC system respectively. The simulation processes of the hybrid simulation are analysed in detail.

The results of hybrid simulation in Matlab and the results of electromagnetic transient model in PSCAD / EMTDC show that the proposed hybrid simulation can reflect accurately the dynamic process of the AC-DC system under different AC-DC system disturbances when the dynamic phasor model of HVDC system which is easier to solve than the EMT model of HVDC system and larger simulation step are used. The simulation results show the accuracy and validity of the hybrid simulation. The proposed hybrid simulation method provides a new method for simulation of AC-DC system.

\section{ACKNOWLEDGEMENTS}

This work was supported by the project of Power Dispatch Control Centre of Guangdong Power Grid Corp.Ltd (KGD2014-198) named "Research on Intelligent Separation Control and Self-Healing Restoration of AC-DC Hybrid Power Systems (Self-Healing Restoration)".

\section{REFERENCES}

[1] Daochun Huang,Yinbiao Shu,Jiangjun Ruan and Yi Hu.Ultra High Voltage Transmission in China:Developments, Current Status and Future Prospects [J].Proceedings of the IEEE,2009,97(3):555-583.

[2] Haojun Zhu,Zexiang Cai,Ani M.Gole,Ming Yu.Improved CoherencyBased Wide-Band Equivalents for Real-Time Digital Simulators [J].IEEE Transaction on Power System,2011,26(3):1410-1417.

[3] Prabha Kunder.Power system stability and control[M].New York:McGraw-Hill, 1994:545-557.

[4] M. P. Bahrman and B. K. Johnson, "The ABCs of HVDC transmission technologies," in IEEE Power and Energy Magazine, vol. 5, no. 2, pp. 32-44, March-April 2007.

[5] Sanders S R,Noworolshi J M,Liu X Z,et al.Generalized averaging method for power conversion circuits[J].IEEE Transactions on Power Electronics, 1991,6(2):251-259.

[6] Chongru Liu,Anjan Bose,Pengfei Tian.Modeling and Analysis of HVDC Converter Three-Phase Dynamic Phasor[J].IEEE Transactions on Power Delivery, 2014,29(1):3-12. 
[7] Qi Qingru,Jiao Lianwei,Cheng Shousun,et al.Modeling and Simulation of HVDC with Dynamic Phasors[J].Proceedings of the CSEE,2003,23(12):28-32.

8] Fermando Cattan Jusan,Sergio Gomes Jr.,Glauco Nery Taranto.SSR results obtained with a dynamic phasor model of SVC using model analysis[J].Electrical Power and Energy System,2010,26(3):1744-1754.

[9] V. A. Caliskan, O. C. Verghese, and A.M. Stankovic, "Multifrequency averaging of dc-dc converters," IEEE Trans. Power Electronics., vol. 14,no. 1, pp. 124-133, Jan. 1999.

[10] M.Daryabak,S.Filizadeh,J.Jatskvich,et al."Modeling of LCC-HVDC systems using dynamic phasors," "Modeling of LCC-HVDC Systems Using Dynamic Phasors," in IEEE Transactions on Power Delivery, vol. 29, no. 4, pp. 1989-1998

[11] Zhu Haojun,Cai Zexing,Liu Haoming,et al.Hybrid Simulation of AC/DC Power System Based on Dynamic Phasor Model[J].Journal of South China University of Technology(Natural Science Edition), 2006,34(10):83-18.

[12] CHEH Lei,ZHANGKan-jun,XIA Yong-jun,et al.Electromechanicalelectromagnetic transient hybrid simulation on HVDC power transmission system based on ADPSS [J].Power System Protection and Control,2013,41(12):137-142.

[13] Daozhi Xia. Power system analysis [M]. Water Conservancy and Electric Power Press, 1995, 56-59.

[14] J. Ögren,"PLL design for inverter grid connection: Simulations for ideal and non-ideal grid conditions",2010, [online] Available: http://www.divaportal.org/smash/record.jsf?pid=diva2:430787. 